OPEN ACCESS

Edited and reviewed by:

Pietro Genovese,

Boston Children's Hospital and

Harvard Medical School,

United States

*Correspondence:

Mario Amendola

mamendola@genethon.fr

${ }^{\dagger}$ Present address: Giulia Pavani,

The Children's Hospital of Philadelphia, Raymond G. Perelman

Center for Cellular and Molecular

Therapeutics, Philade/phia, PA,

United States

Specialty section: This article was submitted to Genome Editing in Blood Disorders, a section of the journal

Frontiers in Genome Editing

Received: 17 March 2021 Accepted: 06 April 2021

Published: 17 May 2021

Citation:

Pavani G and Amendola M (2021) Corrigendum: Targeted Gene Delivery:

Where to Land

Front. Genome Ed. 3:682171. doi: 10.3389/fgeed.2021.682171

\section{Corrigendum: Targeted Gene Delivery: Where to Land}

\author{
Giulia Pavani ${ }^{\dagger}$ and Mario Amendola* \\ INTEGRARE, UMR_S951, Genethon, Inserm, Univ Evry, Univ Paris-Saclay, Evry, France
}

Keywords: genome editing, gene therapy, nuclease, CRISPR, targeted integration (TI), knock-in, safe harbor, homologous recombination (HR)

\section{A Corrigendum on}

Targeted Gene Delivery: Where to Land

by Pavani, G., and Amendola, M. (2021). Front. Genome Ed. 2:609650. doi: $10.3389 /$ fgeed.2020.609650

In the original article, there was a mistake in Table $\mathbf{1}$ as published. The references indicated in row $\mathbf{B}$ are wrong. The corrected Table $\mathbf{1}$ appears in the attached below.

The authors apologize for this error and state that this does not change the scientific conclusions of the article in any way. The original article has been updated. 
TABLE 1 | (A-F) The advantages and disadvantages of different integration strategies.

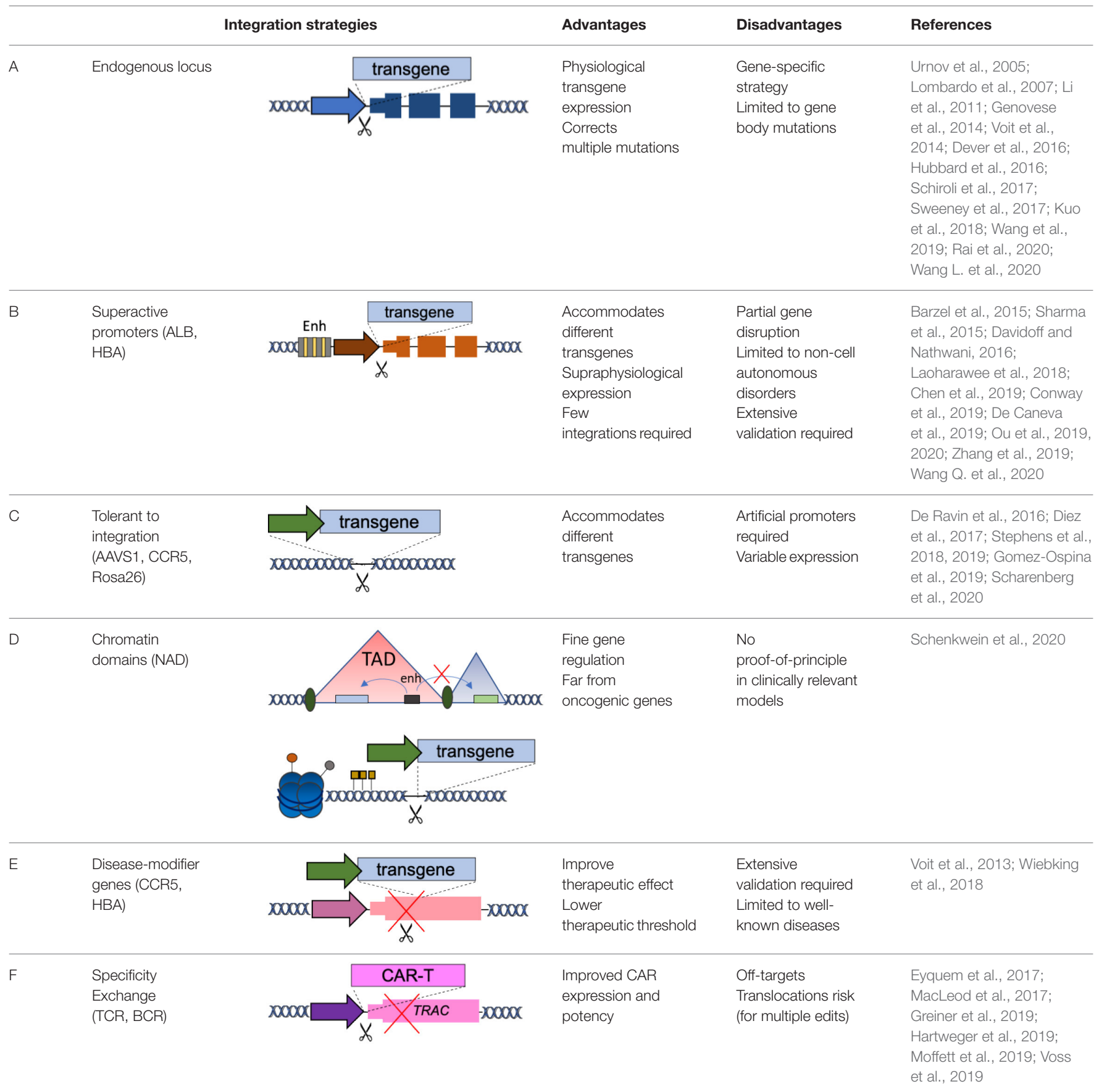

Scissors: nuclease; Solid arrows: promoters; Enh, enhancers; TAD, topologically associating; d, domain; Solid ovals: histone modifications; Solid squares: DNA modifications.

\section{REFERENCES}

Barzel, A., Paulk, N. K., Shi, Y., Huang, Y., Chu, K., Zhang, F., et al. (2015). Promoterless gene targeting without nucleases ameliorates haemophilia B in mice. Nature 517, 360-364. doi: 10.1038/nature13864

Chen, H., Shi, M., Gilam, A., Zheng, Q., Zhang, Y., Afrikanova, I., et al. (2019). Hemophilia A ameliorated in mice by CRISPR-based in vivo genome editing of human factor. Sci. Rep. 9:16838. doi: 10.1038/s41598-019-53198-y

Conway, A., Mendel, M., Kim, K., McGovern, K., Boyko, A., Zhang, L., et al. (2019). Non-viral delivery of zinc finger nuclease mRNA enables highly efficient in vivo genome editing of multiple therapeutic gene targets. Mol. Ther. 27, 866-877. doi: 10.1016/j.ymthe.2019.0 3.003

Davidoff, A. M., and Nathwani, A. C. (2016). Genetic targeting of the albumin locus to treat Hemophilia. N. Engl. J. Med. 374, 1288-1290. doi: 10.1056/NEJMcibr1600347

De Caneva, A., Porro, F., Bortolussi, G., Sola, R., Lisjak, M., Barzel, A., et al. (2019). Coupling AAV-mediated promoterless gene targeting to SaCas9 nuclease to efficiently correct liver metabolic diseases. JCI Insight. 5:128863. doi: $10.1172 /$ jci.insight. 128863 
De Ravin, S. S., Reik, A., Liu, P. Q., Li, L., Wu, X., Su, L., et al. (2016). Targeted gene addition in human CD34(+) hematopoietic cells for correction of X-linked chronic granulomatous disease. Nat. Biotechnol. 34, 424-429. doi: $10.1038 /$ nbt.3513

Dever, D. P., Bak, R. O., Reinisch, A., Camarena, J., Washington, G., Nicolas, C. E., et al. (2016). CRISPR/Cas9 beta-globin gene targeting in human haematopoietic stem cells. Nature 539, 384-389. doi: 10.1038/nature20134

Diez, B., Genovese, P., Roman-Rodriguez, F. J., Alvarez, L., Schiroli, G., Ugalde, L., et al. (2017). Therapeutic gene editing in CD34(+) hematopoietic progenitors from Fanconi anemia patients. EMBO Mol. Med. 9, 1574-1588. doi: 10.15252/emmm.201707540

Eyquem, J., Mansilla-Soto, J., Giavridis, T., van der Stegen, S. J., Hamieh, M., Cunanan, K. M., et al. (2017). Targeting a CAR to the TRAC locus with CRISPR/Cas9 enhances tumour rejection. Nature 543, 113-117. doi: 10.1038/nature21405

Genovese, P., Schiroli, G., Escobar, G., Tomaso, T. D., Firrito, C., Calabria, A., et al. (2014). Targeted genome editing in human repopulating haematopoietic stem cells. Nature 510, 235-240. doi: 10.1038/nature 13420

Gomez-Ospina, N., Scharenberg, S. G., Mostrel, N., Bak, R. O., Mantri, S., Quadros, R. M., et al. (2019). Human genome-edited hematopoietic stem cells phenotypically correct Mucopolysaccharidosis type. Nat. Commun. 10:4045. doi: 10.1038/s41467-019-11962-8

Greiner, V., Bou Puerto, R., Liu, S., Herbel, C., Carmona, E. M., and Goldberg, M. S. (2019). CRISPR-mediated editing of the B cell receptor in primary human B cells. iScience 12, 369-378. doi: 10.1016/j.isci.2019.01.032

Hartweger, H., McGuire, A. T., Horning, M., Taylor, J. J., Dosenovic, P., Yost, D., et al. (2019). HIV-specific humoral immune responses by CRISPR/Cas9-edited B cells. J. Exp. Med. 216, 1301-1310. doi: 10.1084/jem.20190287

Hubbard, N., Hagin, D., Sommer, K., Song, Y., Khan, I., Clough, C., et al. (2016). Targeted gene editing restores regulated CD40L function in X-linked hyperIgM syndrome. Blood 127, 2513-2522. doi: 10.1182/blood-2015-11-683235

Kuo, C. Y., Long, J. D., Campo-Fernandez, B., de Oliveira, S., Cooper, A. R., Romero, Z., et al. (2018). Site-specific gene editing of human hematopoietic stem cells for X-linked hyper-IgM syndrome. Cell Rep. 23, 2606-2616. doi: 10.1016/j.celrep.2018.04.103

Laoharawee, K., DeKelver, R. C., Podetz-Pedersen, K. M., Rohde, M., Sproul, S., Nguyen, H. O., et al. (2018). Dose-dependent prevention of metabolic and neurologic disease in murine MPS II by ZFN-mediated in vivo genome editing. Mol. Ther. 26, 1127-1136. doi: 10.1016/j.ymthe.2018.03.002

Li, H., Haurigot, V., Doyon, Y., Li, T., Wong, S. Y., Bhagwat, A. S., et al. (2011). In vivo genome editing restores haemostasis in a mouse model of haemophilia. Nature 475, 217-221. doi: 10.1038/nature10177

Lombardo, A., Genovese, P., Beausejour, C. M., Colleoni, S., Lee, Y. L., Kim, K. A., et al. (2007). Gene editing in human stem cells using zinc finger nucleases and integrase-defective lentiviral vector delivery. Nat. Biotechnol. 25, 1298-1306. doi: $10.1038 /$ nbt1353

MacLeod, D. T., Antony, J., Martin, A. J., Moser, R. J., Hekele, A., Wetzel, K. J., et al. (2017). Integration of a CD19 CAR into the TCR alpha chain locus streamlines production of allogeneic gene-edited CAR T cells. Mol. Ther. 25, 949-961. doi: 10.1016/j.ymthe.2017.02.005

Moffett, H. F., Harms, C. K., Fitzpatrick, K. S., Tooley, M. R., Boonyaratanakornkit, J., and Taylor, J. J. (2019). B cells engineered to express pathogenspecific antibodies protect against infection. Sci. Immunol. 4:aax0644. doi: 10.1126/sciimmunol.aax0644

Ou, L., DeKelver, R. C., Rohde, M., Tom, S., Radeke, R., St Martin, S. J., et al. (2019). ZFN-mediated in vivo genome editing corrects murine hurler syndrome. Mol. Ther. 27, 178-187. doi: 10.1016/j.ymthe.2018.10.018

Ou, L., Przybilla, M. J., Ahlat, O., Kim, S., Overn, P., Jarnes, J., et al. (2020). A highly efficacious PS gene editing system corrects metabolic and neurological complications of Mucopolysaccharidosis type I. Mol. Ther. 28, 1442-1454. doi: 10.1016/j.ymthe.2020.03.018

Rai, R., Romito, M., Rivers, E., Turchiano, G., Blattner, G., Vetharoy, W., et al. (2020). Targeted gene correction of human hematopoietic stem cells for the treatment of Wiskott - Aldrich Syndrome. Nat. Commun. 11:4034. doi: 10.1038/s41467-020-17626-2

Scharenberg, S. G., Poletto, E., Lucot, K. L., Colella, P., Sheikali, A., Montine, T. J., et al. (2020). Engineering monocyte/macrophage-specific glucocerebrosidase expression in human hematopoietic stem cells using genome editing. Nat. Commun. 11:3327. doi: 10.1038/s41467-020-17148-x

Schenkwein, D., Afzal, S., Nousiainen, A., Schmidt, M., and YlaHerttuala, S. (2020). Efficient nuclease-directed integration of lentivirus vectors into the human ribosomal DNA locus. Mol. Ther. 28, 1858-1875. doi: 10.1016/j.ymthe.2020.0 5.019

Schiroli, G., Ferrari, S., Conway, A., Jacob, A., Capo, V., Albano, L., et al. (2017). Preclinical modeling highlights the therapeutic potential of hematopoietic stem cell gene editing for correction of SCID-X1. Sci. Transl. Med. 9:aan0820. doi: 10.1126/scitranslmed.aan0820

Sharma, R., Anguela, X. M., Doyon, Y., Wechsler, T., DeKelver, R. C., Sproul, S., et al. (2015). In vivo genome editing of the albumin locus as a platform for protein replacement therapy. Blood 126, 1777-1784. doi: 10.1182/blood-2014-12-61 5492

Stephens, C. J., Kashentseva, E., Everett, W., Kaliberova, L., and Curiel, D. T. (2018). Targeted in vivo knock-in of human alpha-1-antitrypsin cDNA using adenoviral delivery of CRISPR/Cas9. Gene Ther. 25, 139-156. doi: 10.1038/s41434-018-0003-1

Stephens, C. J., Lauron, E. J., Kashentseva, E., Lu, Z. H., Yokoyama, W. M., and Curiel, D. T. (2019). Long-term correction of hemophilia B using adenoviral delivery of CRISPR/Cas9. J. Contr. Release. 298, 128-141. doi: 10.1016/j.jconrel.2019.02.009

Sweeney, C. L., Zou, J., Choi, U., Merling, R. K., Liu, A., Bodansky, A., et al. (2017). Targeted repair of CYBB in X-CGD iPSCs requires retention of intronic sequences for expression and functional correction. Mol. Ther. 25, 321-330. doi: 10.1016/j.ymthe.2016.11.012

Urnov, F. D., Miller, J. C., Lee, Y. L., Beausejour, C. M., Rock, J. M., Augustus, S., et al. (2005). Highly efficient endogenous human gene correction using designed zinc-finger nucleases. Nature 435, 646-651. doi: 10.1038/nature03556

Voit, R. A., Hendel, A., Pruett-Miller, S. M., and Porteus, M. H. (2014). Nuclease-mediated gene editing by homologous recombination of the human globin locus. Nucl. Acids Res. 42, 1365-1378. doi: 10.1093/nar/gk t947

Voit, R. A., McMahon, M. A., Sawyer, S. L., and Porteus, M. H. (2013). Generation of an HIV resistant T-cell line by targeted "stacking" of restriction factors. Mol. Ther. 21, 786-795. doi: 10.1038/mt.2012.284

Voss, J. E., Gonzalez-Martin, A., Andrabi, R., Fuller, R. P., Murrell, B., McCoy, L. E., et al. (2019). Reprogramming the antigen specificity of B cells using genome-editing technologies. Elife 8:42995. doi: 10.7554/eLife.42995

Wang, L., Yang, Y., Breton, C., Bell, P., Li, M., Zhang, J., et al. (2020). A mutation-independent CRISPR-Cas9-mediated gene targeting approach to treat a murine model of ornithine transcarbamylase deficiency. Sci. Adv. 6:eaax5701. doi: 10.1126/sciadv.aax5701

Wang, L., Yang, Y., Breton, C. A., White, J., Zhang, J., Che, Y., et al. (2019). CRISPR/Cas9-mediated in vivo gene targeting corrects hemostasis in newborn and adult factor IX-knockout mice. Blood 133, 2745-2752. doi: 10.1182/blood.2019000790

Wang, Q., Zhong, X., Li, Q., Su, J., Liu, Y., Mo, L., et al. (2020). CRISPR-Cas9mediated in vivo gene integration at the albumin locus recovers hemostasis in neonatal and adult hemophilia B mice. Mol. Ther. Methods Clin. Dev. 18, 520-531. doi: 10.1016/j.omtm.2020.06.025

Wiebking, V., Patterson, J. O., Martin, R., Chanda, M. K., Lee, C. M., Srifa, W., et al. (2018). Metabolic engineering generates a transgene-free safety switch for cell therapy. Nat. Biotechnol 2020:6. doi: 10.1038/s41587-020-0580-6

Zhang, J. P., Cheng, X. X., Zhao, M., Li, G. H., Xu, J., Zhang, F., et al. (2019). Curing hemophilia A by NHEJ-mediated ectopic F8 insertion in the mouse. Genome Biol. 20:276. doi: 10.1186/s13059-019-1907-9

Copyright (c) 2021 Pavani and Amendola. This is an open-access article distributed under the terms of the Creative Commons Attribution License (CC BY). The use, distribution or reproduction in other forums is permitted, provided the original author(s) and the copyright owner(s) are credited and that the original publication in this journal is cited, in accordance with accepted academic practice. No use, distribution or reproduction is permitted which does not comply with these terms. 